\title{
Early growth response protein 1 acts as an activator of SOX18 promoter
}

\author{
Isidora Petrovic, Natasa Kovacevic-Grujicic \\ and Milena Stevanovic ${ }^{1}$
}

\author{
Institute of Molecular Genetics and Genetic Engineering \\ University of Belgrade \\ 11010 Belgrade, Serbia \\ ${ }^{1}$ Corresponding author: Tel, 381-113976212; \\ Fax, 381-113975808; E-mail, stevanov@eunet.rs \\ DOI 10.3858/emm.2010.42.2.015
}

Accepted 7 December 2009

Available Online 7 January 2010

Abbreviations: CAT, chloramphenicol acetyltransferase; EGR1, early growth response 1; EMSA, electrophoretic mobility shift assay; HMG, high mobility group box; NF-Y, nuclear factor $Y$; SOX, sex-determining region $Y$ box; Sp3, specificity protein 3 ; tsp, transcription start point; WCL, whole cell lysates; ZBP-89, zinc finger binding protein 89

\begin{abstract}
Sex-determining region $\mathrm{Y}$ box 18 (Sox18/SOX18) gene is an important regulator of vascular development playing a role in endothelial cell specification or differentiation, angiogenesis and atherogenesis. The aim of this study was to perform comprehensive functional characterization of the human SOX18 promoter, including determination of transcription start point ( $t s p$ ) and identification of control elements involved in the regulation of SOX18 gene expression, with an emphasis on angiogenesis-related transcription factors. Analyses were performed in HeLa cells, representing a tumor cell line, and in EA.hy926 cells used as an endothelial model system. We have determined unique $t s p$ of SOX18gene, located 172 nucleotides upstream from ATG codon. Further, we have shown that SOX18 promoter region, $-\mathbf{7 2 6}$ to -89 bp relative to $t s p$, contains positive cis-regulatory element(s) that stimulates SOX18 promoter activity, while region -89 to +166 represents the minimal promoter. Within this region we have recognized the presence of essential element(s), positioned from -89 to +29 , which harbors cluster of three putative early growth response 1 (EGR1) binding sites. By in vitro binding assays and functional analyses we have shown that these three putative binding sites are functionally relevant and sufficient for
\end{abstract}

EGR1-induced SOX18 transcription. Mutations of these binding sites significantly impaired activity of the SOX18 promoter, particularly in EA.hy926 cells, indicating the importance of these regulatory elements for SOX18 promoter activity in endothelial setting. By data presented in this study, we have established SOX18 as a novel target gene regulated by EGR1 transcription factor, thus providing the first functional link between two transcription factors previously shown to be involved in the control of angiogenesis.

Keywords: early growth response protein 1; endothelial cells; neovascularization, physiologic; promoter regions, genetic; SOX18 protein, human; transcription, genetic

\section{Introduction}

Sex-determining region $Y$ box (SOX) genes constitute a large family of diverse and well-conserved genes encoding transcription factors implicated in the control of various developmental processes (Pevny and Lovell-Badge, 1997; Wegner, 1999). SOX proteins contain a characteristic DNA-binding motif commonly known as the high mobility group domain (HMG domain) and display properties of both classical transcription factors and architectural components of chromatin (Pevny and LovellBadge, 1997). Based on homologies, both within and outside of the HMG box, this gene family can be subdivided into ten groups, A to J (Bowles et al., 2000). Sox18/SOX18 gene, together with Sox7/ SOX7 and Sox17/SOX17 genes, belongs to SOX group F. Previously, it has been shown that Sox group $F$ genes have a role in vascular development and postnatal neovascularization (Matsui et al., 2006; Cermenati et al., 2008). The functional importance of SOX18 protein in vascular development is revealed by the vascular defects caused by Sox18/SOX18 mutations in mice and humans. Mutations in Sox18 underlie the mutant phenotype of ragged mutant mouse (Downes and Koopman, 2001) and mutations in human SOX18 are associated with hypotrichosis-lymphedema-telangiectasia syndrome (Irrthum et al., 2003). Murine Sox18 is demonstrated to be involved in the induction of angiogenesis during wound healing and tissue repair (Darby et al., 2001) and SOX18 is shown to play a role in atherosclerosis in humans (Garcia- 


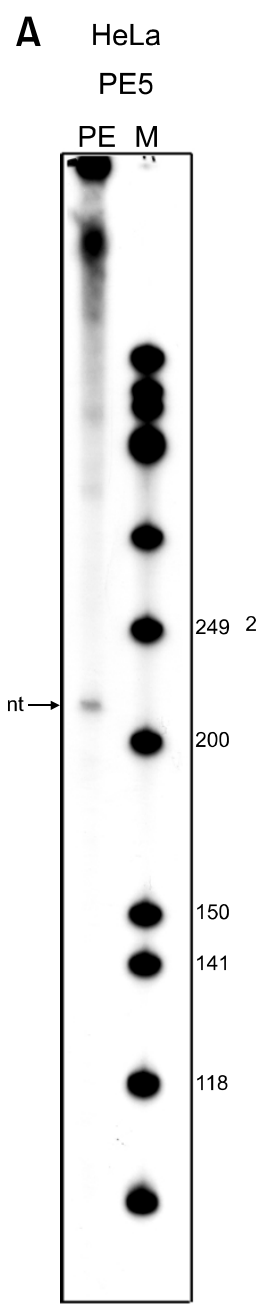

B EA.hy926

PE5

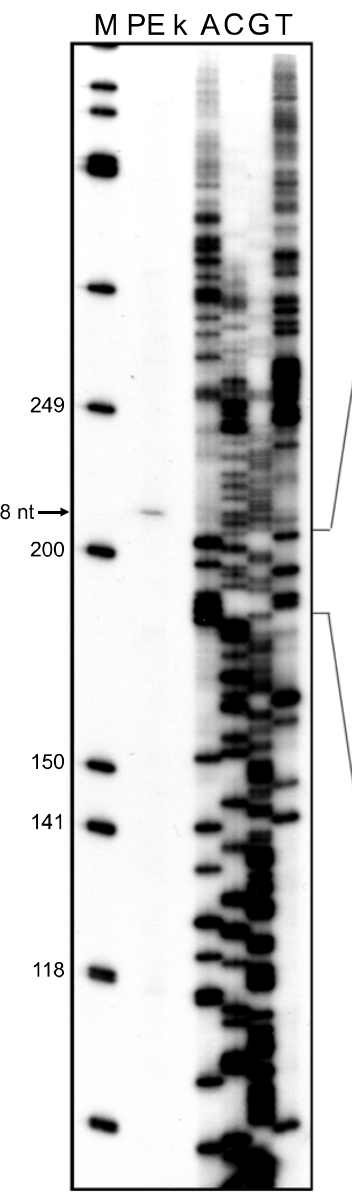

C EA.hy926

PE3

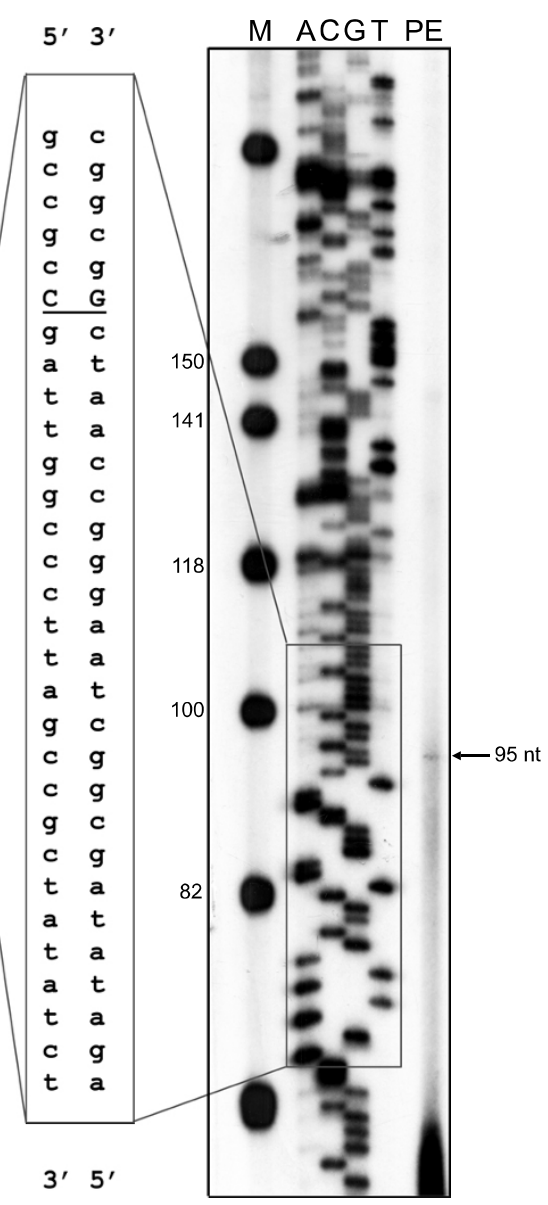

Figure 1. Mapping of the SOX 18 tsp by primer extension analysis. Total RNA from HeLa (A) and EA.hy926 cells $(B, C)$ were hybridized to the radiolabeled antisense primers PE5 (A, B) and PE3 (C), and primer extension reactions were performed as described in Methods. Control reaction was performed with yeast tRNA ( $B$, lane $k$ ). The extension products are marked by arrows (A, B, C, lanes PE). The radiolabeled $P$ hiX/Hinflll digest was used as a ladder (A, B, C, lane $M$ ). Lanes ACGT indicate the nucleotide sequence generated with the corresponding primers. The DNA sequence is shown with first nucleotide of the transcript underlined and presented in capital case.
Ramirez et al., 2005). Furthermore, it has been demonstrated that interfering with SOX18 function inhibits blood vessel formation and subsequent tumor growth (Young et al., 2006).

Despite the mounting evidence that SOX18 protein is an important player in vascular development, atherogenesis and angiogenesis, little is known about transcription factors involved in tissue and cell-type specific regulation of its expression. So far, we have characterized the SOX18 promoter region and demonstrated that ubiquitous transcription factors specificity protein 3 (Sp3), zinc finger binding protein 89 (ZBP-89) and nuclear factor $Y$ (NF-Y) are involved in the regulation of its expression in HeLa tumor cell line (Petrovic and Stevanovic, 2007; Petrovic et al., 2009). Here, we have been particularly interested in discovering angiogenesis-related transcription factor(s) that might have the role in regulation of SOX18 gene expression in endothelial setting.

It has been shown that early growth response protein 1 (EGR1) plays a pivotal role in the transcriptional response of endothelial cells to angiogenic growth factors involved in angiogenic switch (Lucerna et al., 2006). Namely, EGR1, a zinc-finger transcription factor that binds to GC-rich cis-acting promoter elements controls the expression of a wide variety of pathogenesis-relevant genes, including growth factors, cytokines and receptors, with many of them involved in angiogenesis and tumorigenesis (Fahmy et al., 2003; Lucerna et al., 2003). There is a number of genes identified as possible EGR1 targets and they play functional roles in normal development and differentiation, as well as, in the various pathological settings (Silverman and Collins, 1999).

In this report, we have presented data demonstrating transcriptional up-regulation of the human SOX18 gene by EGR1 in tumor (HeLa) and endothelial (EA.hy926) cells. EA.hy926 is a permanent human cell line derived from human umbilical vein endothelial cell (HUVEC) that displays a number of 
features characteristic for vascular endothelial cells (Edgell et al., 1983). Therefore, these cells were used as an endothelial model system in the present study.

Presented results provide the first functional link between SOX18 and EGR1, previously shown to be involved in the regulation of angiogenesis. Understanding of molecular mechanisms involved in human SOX18 gene transcriptional regulation could help in better understanding of important physiological and patho-physiological processes in which SOX18 participates.

\section{Results}

\section{Mapping of the SOX18 transcription start point}

The first step in functional characterization of SOX18 promoter was to determine the tsp of the human SOX18 gene. For that purpose we have performed primer extension analysis, using total RNA isolated from HeLa and EA.hy926 cells. Single products were obtained with the length of 208 nucleotides and 95 nucleotides with PE5 and PE3 primers, respectively (Figures $1 \mathrm{~A}, 1 \mathrm{~B}$ and $1 \mathrm{C}$ ). Comparison to dideoxy-sequencing reaction revealed that both primers extended to the same cytosine residue, 172 nucleotides upstream from ATG codon and this nucleotide was designated as +1 (Figures $1 \mathrm{~B}$ and $1 \mathrm{C}$ ). These results demonstrate that SOX18 transcription initiates at the single major site, regardless of different origin of $\mathrm{HeLa}$ and EA.hy926 cells. The position of tsp, determined by this analysis, correlates with the length of SOX18 transcript that we have detected earlier by Northern blotting (Petrovic and Stevanovic, 2007).

\section{Transcriptional activity of SOX18 promoter deletion constructs}

Previously, we demonstrated that SOX18 5' flanking fragment $892 \mathrm{bp}$ in size upstream from the start codon represents SOX18 promoter region (Petrovic and Stevanovic, 2007). Here, the same regulatory region, together with its three different deletion fragments, has been subjected to further functional analysis in HeLa and EA.hy926 cells (Figure 2A). As presented on Figure 2B, the full length promoter construct (892pCAT6) displayed maximum reporter activity, while 5 ' deletion up to -89 (255pCAT6) caused decrease of CAT activity to approximately $30 \%$ and $60 \%$ in HeLa and EA.hy 926 cells, respectively. These results indicate that SOX18 promoter region, spanning the sequence -726 to $-89 \mathrm{bp}$ relative to $t s p$, contains positive cis-regulatory element(s) responsible for
A

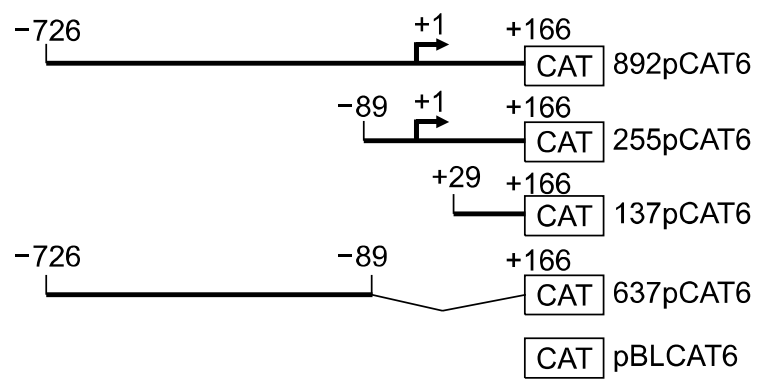

B
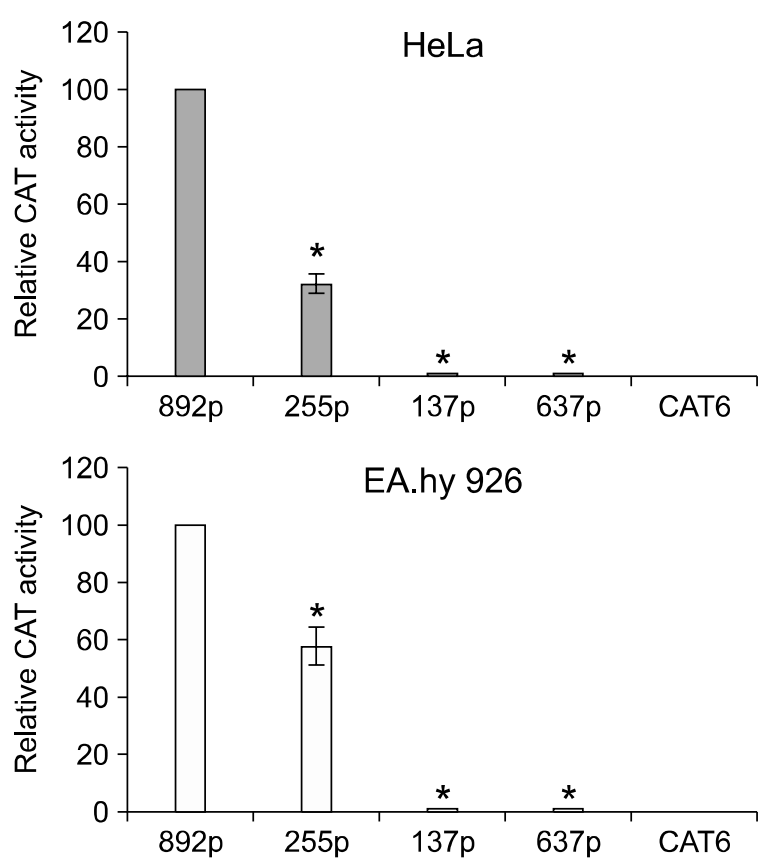

Figure 2. Functional analysis of the human SOX18 promoter. (A) Schematic representation of the cat reporter constructs. Numbers indicate the positions of fragments end points relative to the tsp. (B) Reporter activities of the SOX18 promoter constructs in HeLa cells (grey bars) and EA.hy926 (white bars). The normalized CAT activities were evaluated as a percentage of the $892 \mathrm{pCAT} 6$ construct, which was set as $100 \%$ and are presented as the means \pm SEM of at least five independent experiments. Values of $P<0.001$ are presented by *.

stimulation of SOX18 promoter activity. Further 5' deletion up to +29 (construct 137 pCAT6) resulted in complete abolition of the promoter activity, thus revealing the presence of essential element(s) positioned between -89 and +29 relative to tsp (Figure 2B). Additionally, 3' deletion of the full length construct up to -89 (construct 637 pCAT6) caused complete loss of promoter activity, indicating that region -89 to $+166 \mathrm{bp}$ relative to $t s p$ (construct 255pCAT6) harbors regulatory elements indispensable for SOX18 transcription in both cell lines and represents the minimal promoter region 
A

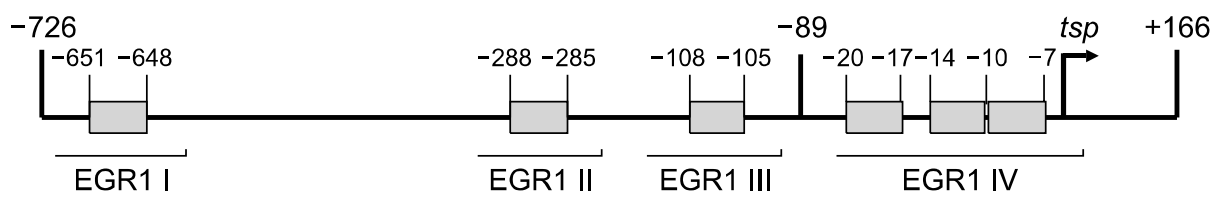

B

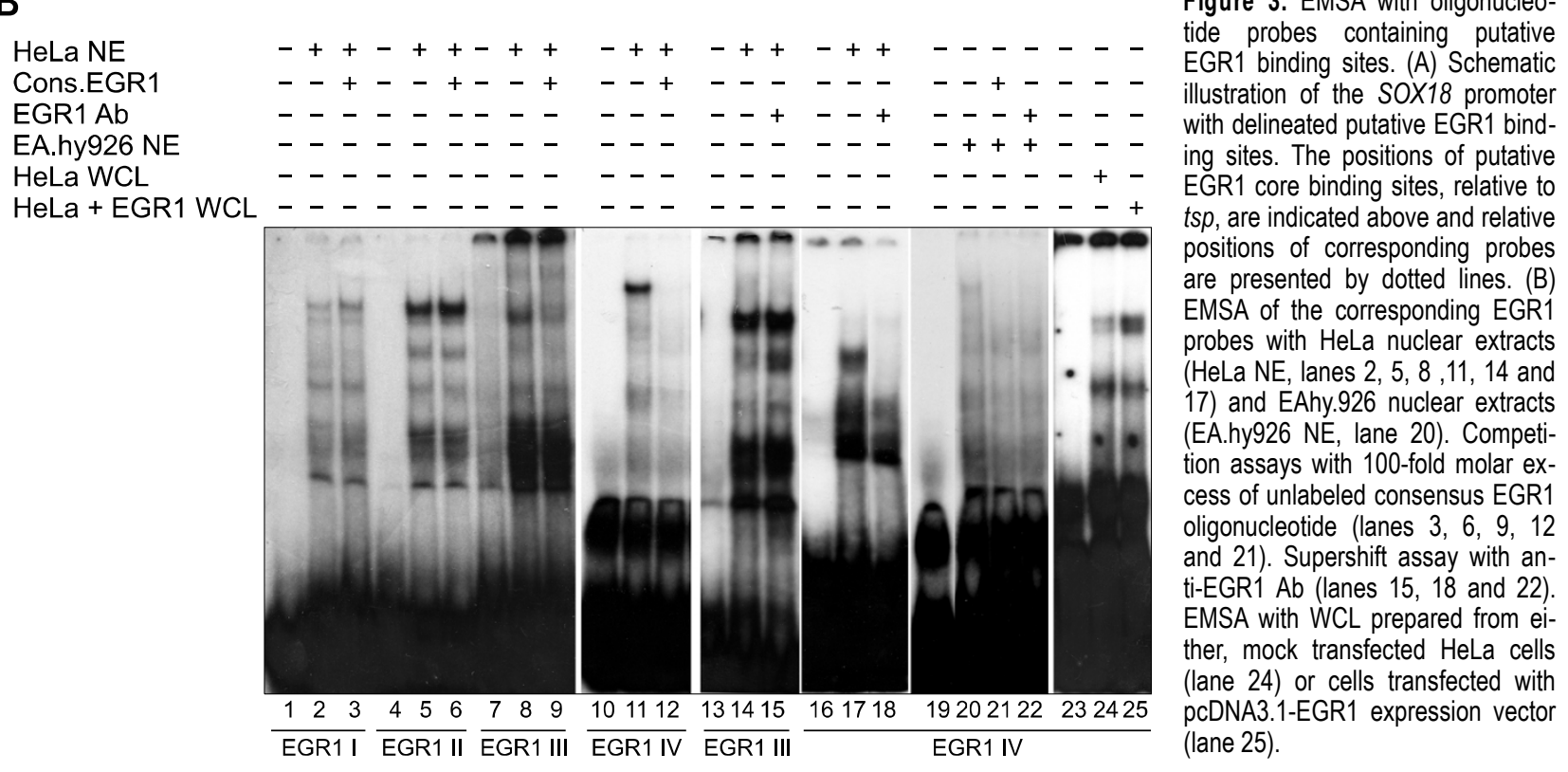

of the SOX18 gene.

\section{EGR1 binds within SOX18 minimal promoter region in vitro}

Using in silico analysis, within SOX18 promoter region, we have identified a plethora of putative binding sites for various, mainly ubiquitous transcription factors (data not shown). Among them, we have identified six putative binding sites for angiogenesis-related, zing-finger transcription factor EGR1 (Figure 3A). As shown on Figure 3A, three putative EGR1 binding sites are located within the region -726 to $-89 \mathrm{bp}$ relative to $t s p$, and another three are clustered within minimal promoter region immediately upstream of the $t s p$. In order to determine to which of these putative binding sites EGR1 binds, we have performed EMSA with four SOX18 DNA probes that harbor different putative EGR1 binding sites (designated as probes EGR1 I to IV, Figure $3 \mathrm{~A}$ ). Nuclear proteins isolated from HeLa cells bind to all four DNA probes and form DNAprotein complexes (Figure 3B, lanes 2, 5, 8 and 11). In competition reaction, EGR1 consensus oligonucleotide probe was used in order to elucidate whether EGR1 participates in DNA-protein com- plex formation. In competition reaction with 100fold molar excess of unlabeled EGR1 consensus probe, we observed complete fading of DNA-protein complexes formed by EGR1 IV probe (Figure 3B, lane 12), moderate competition with EGR1 III probe (Figure 3B, lane 9), and no competition with other two probes (Figures 3B, lanes 3 and 6 ). Accordingly, supershift assays were performed with probes EGR1 III and EGR1 IV only. After addition of anti-EGR1 antibody no supershift or fading of complexes were observed with EGR1 III probe (Figure 3B, lane 15), while significant fading of slowest DNA-protein migrating complex was observed with EGR1 IV probe (Figure 3B, lane 18). Therefore, we extended our analyses with EGR1 IV probe, using nuclear proteins from EA.hy926 cells (Figure 3B, lanes 19 to 22). Both competition with EGR1 consensus probe and addition of anti-EGR1 antibody caused fading of DNA-protein complexes (Figure 3B, lanes 21 and 22, respectively). Fading of DNA-protein complexes in supershift reaction has already been shown in several reports and was considered as a confirmation of specific protein presence (Argyropoulos et al., 2003; Kovacevic Grujicic et al., 2005). In order to further validate obtained results, we have per- 
A

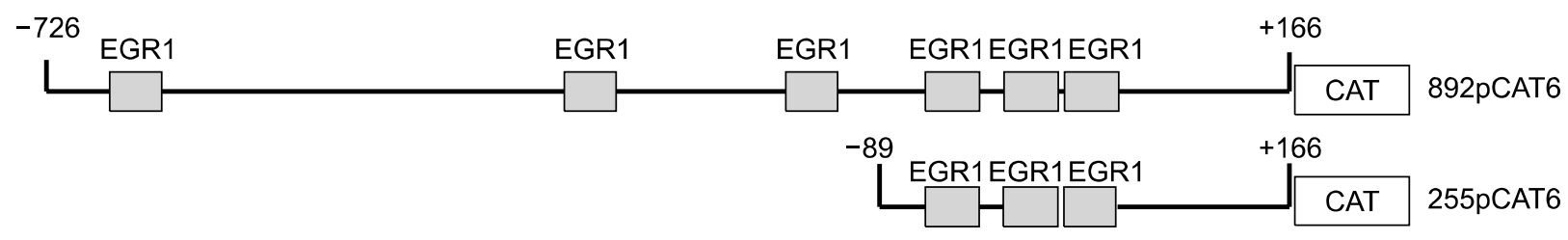

B
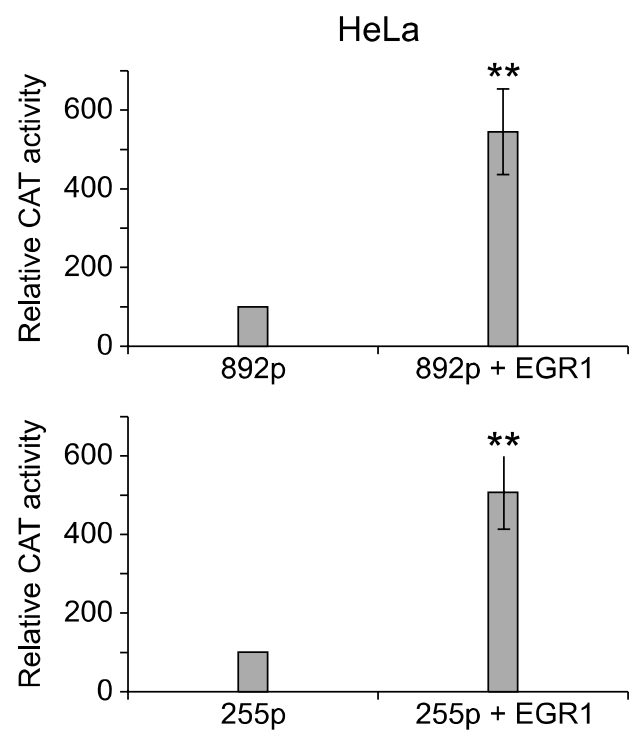

D

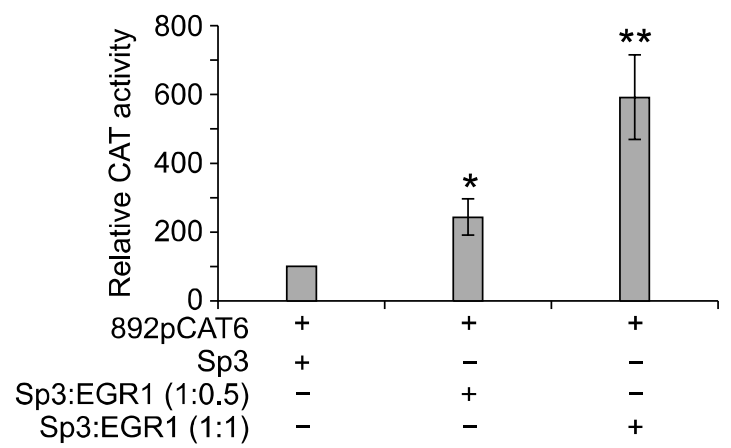

C
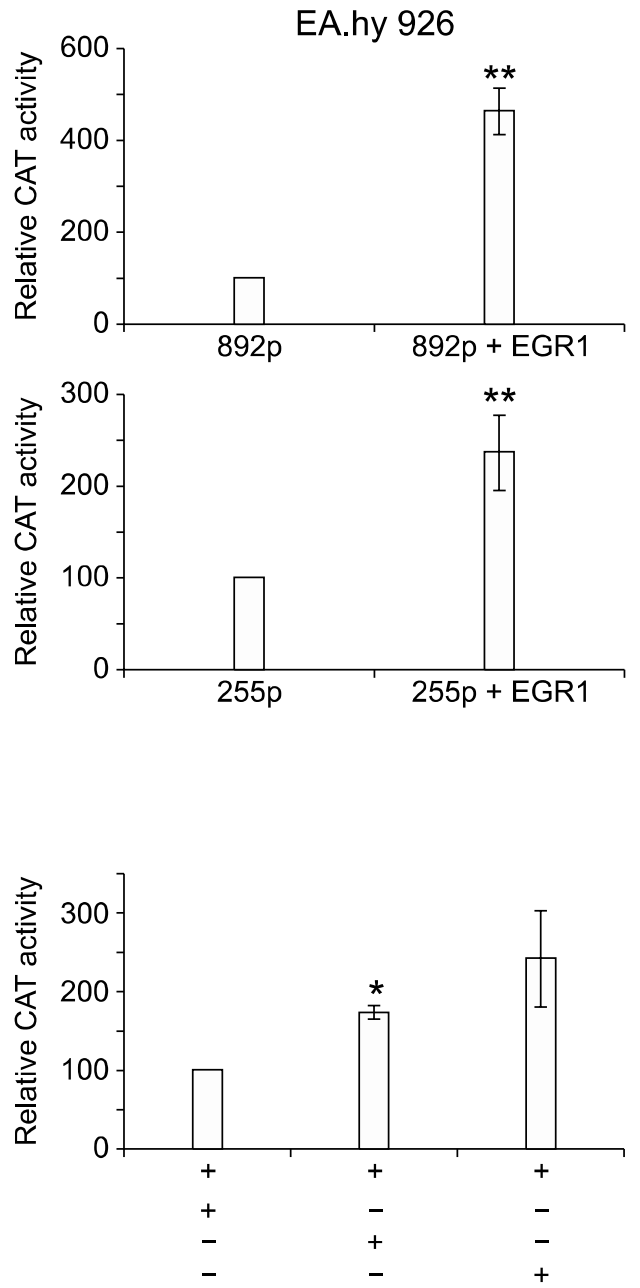

Figure 4. Effects of EGR1 overexpression on SOX18 promoter activity. (A) Schematic illustration of the 892pCAT6 and 255pCAT6 promoter reporter constructs. Grey boxes represent putative EGR1 binding sites within SOX18 promoter region. HeLa cells (B) and EA.hy926 cells (C) were transiently co-transfected with either 892pCAT6 or 255pCAT6 promoter constructs, together with either empty or EGR1 expression vector. Normalized CAT activities were calculated as percentages of the corresponding reporter construct activity in cells co-transfected with empty pcDNA3.1 (which was set as 100\%). Relative CAT activities are presented as the means \pm SEM of three independent experiments. Values of $P<0.05$ are presented by**. (D) Functional competition between Sp3 and EGR1 performed with 892pCAT6 promoter construct. HeLa and EA.hy926 cells were co-transfected with fixed amount of Sp3 expression vector and increasing amounts of EGR1 expression vector, as indicated. Normalized CAT activities were calculated as percentages of the corresponding reporter construct activity in cells co-transfected with Sp3 (which was set as 100\%). Relative CAT activities are presented as the means \pm SEM of at least three independent experiments. Values of $P<0.001$ are presented by* and $P<0.05$ are presented by*

formed EMSA reaction with proteins isolated from HeLa cells transfected with EGR1 expression vector. Compared to the binding of proteins isolated from mock transfected HeLa cells, we have detected increased binding of proteins when exogenous EGR1 is overexpressed (Figure 3B, lanes 24 and 
25). This result demonstrated that EGR1 overexpression significantly increased cellular protein binding to specific SOX18 oligonucleotide probe, indicating its participation in DNA-protein complex formation.

Taken together, we have shown that EGR1 transcription factor specifically binds to the sequence -29 to +10 relative to tsp (EGR1 IV probe), within SOX18 minimal promoter region.

\section{EGR1 up-regulates SOX18 promoter activity in Hela and EA.hy926 cell lines}

In order to explore the role of EGR1 in transcriptional regulation of the human SOX18 gene expression, we have performed transient co-transfection experiments of HeLa and EA.hy926 cells with EGR1 expression vector. We have tested the effect of EGR1 overexpression on the activities of both, full length promoter (construct 892pCAT6) and minimal promoter region (construct 255pCAT6) (Figure 4A). Full length promoter construct (892pCAT6) activity was increased approximately 5 -fold in both HeLa and EA.hy926 cells (Figures $4 \mathrm{~B}$ and $4 \mathrm{C}$ ). Interestingly, response of minimal promoter construct (255pCAT6) to EGR1 overexpression was different between two tested cell lines. Precisely, 255pCAT6 activity was increased approximately 5 -fold in $\mathrm{HeLa}$ and 2.5 -fold in EA.hy926 cells (Figures 4B and 4C).

Thus, in HeLa cell line, EGR1 overexpression up-regulated both promoter constructs to the same extent (Figure 4B). On the other hand, in EA.hy926 cells 2-fold attenuation in the response to EGR1 was observed for construct 255pCAT6, compared to the construct $892 p C A T 6$ (Figure 4C). Nevertheless, these functional overexpression analyses point out that binding sites within minimal promoter region are sufficient for mediating SOX18 transactivation by EGR1 in both model systems used in this study.

We have previously shown that Sp3 transcription factor, an another zinc-finger binding protein, is able to moderately down-regulate SOX18 promoter activity in HeLa cells. Since both Sp3 and EGR1 recognize cluster of overlapping binding sites covered by EGR1 IV probe $(-29$ to +10 relative to tsp) (Figure 3A), we have tested whether EGR1 overexpression could overcome Sp3 inhibitory effect on SOX18 promoter activity. Accordingly, we have performed functional competition assays in which both HeLa and EA.hy926 cells were cotransfected with fixed amount of Sp3 expression vector and increasing amounts of EGR1 expression vector. As presented in Figure 4D, overexpression of EGR1 causes dose dependent re-

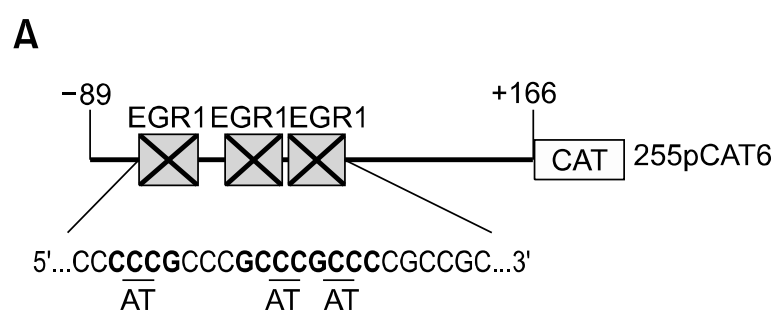

B
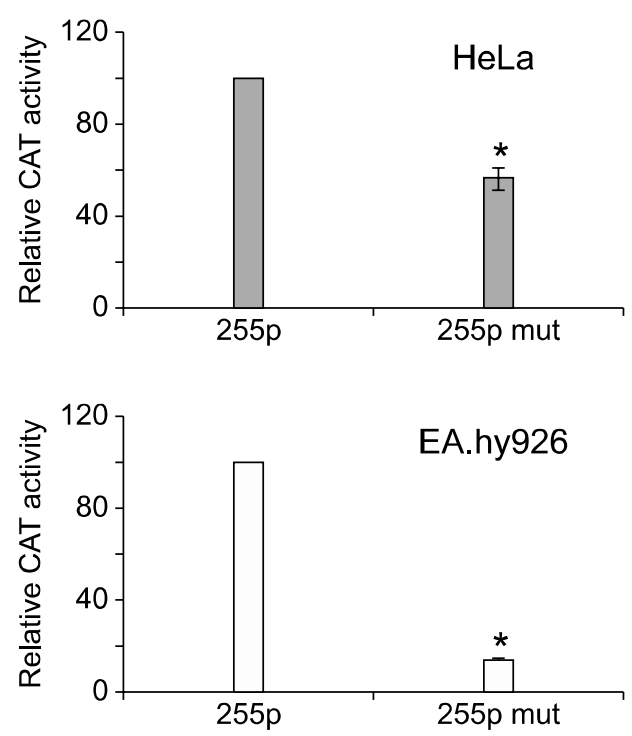

Figure 5. The effect of site directed mutations in EGR1 binding sites. (A) Schematic representation of the mutant construct 255mutCAT6 used in this study. (B) Transient transfection of HeLa and EA.hy926 cells with either wild type 255pCAT6 construct or its mutated counterpart 255mutCAT6. Normalized CAT activities were calculated as percentages of the wild type 255pCAT6 construct activity, which was set as $100 \%$. Relative CAT activities are presented as the means \pm SEM of three independent experiments. Values of $P<0.001$ are presented by*.

activation of SOX18 promoter up to 5.9- and 2.5 -fold in HeLa and EA.hy926 cells, respectively. Our results demonstrate that overexpression of EGR1 transcription factor is capable to overcome Sp3-mediated repression, providing further evidence that EGR1 plays a critical role in transactivation of the SOX18 promoter activity.

\section{Functional analysis of clustered EGR1 binding sites within the human SOX18 minimal promoter region}

In order to further determine the functional role of EGR1 in the regulation of SOX18 expression, we introduced site directed mutations into cluster of three overlapping EGR1 binding sites within construct 255pCAT6 (Figure 5A). The ability of the mutant reporter construct and its wild-type coun- 


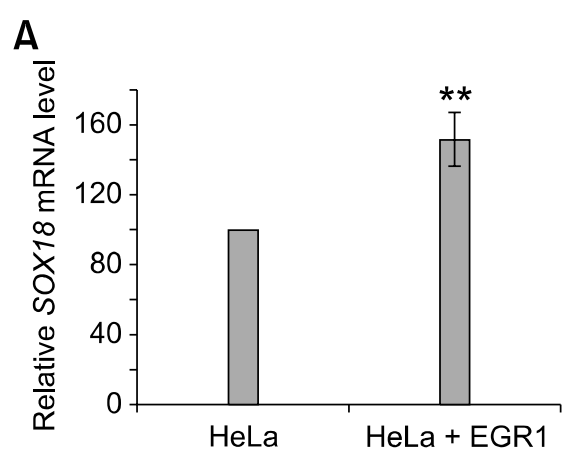

B

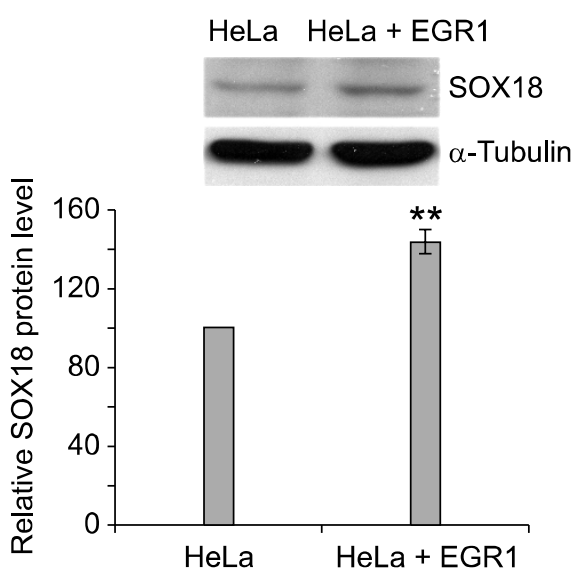

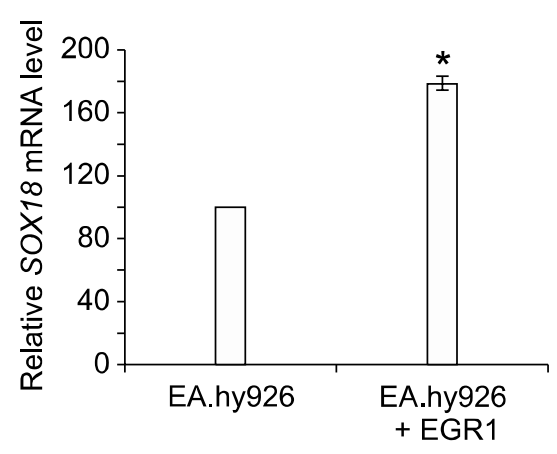

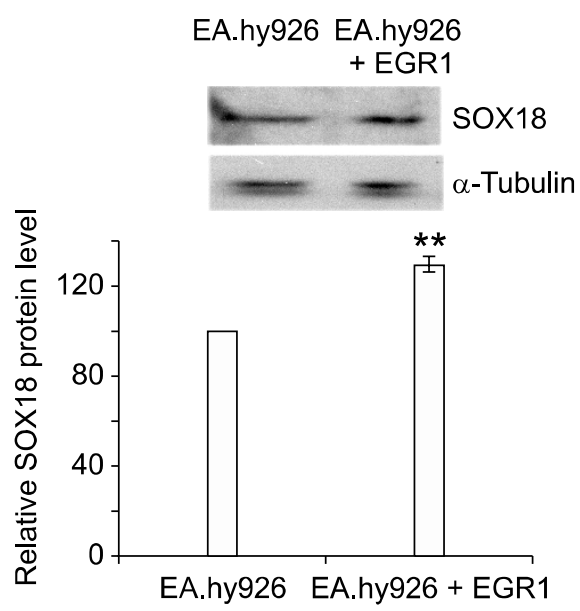

Figure 6. The effect of EGR1 on SOX18 expression in native context. (A) qRT-PCR analysis of SOX18 transcription after transfection of HeLa and EA.hy926 cells with either empty pcDNA3.1 or pcDNA3.1-EGR1 expression vectors. The quantities of SOX18 transcript in cells overexpressing EGR1 were calculated as a percentage of mock transfected cells which was set as $100 \%$. Data of three independent experiments are presented at histograms as the means \pm SEM. Values of ${ }^{* *} P<$ 0.05 are presented by and values of ${ }^{*} P<0.001$ are presented by. (B) Western blot analysis of SOX18 expression after transfection of HeLa and EA.hy926 cells with either empty pcDNA3.1 or pcDNA3.1-EGR1 expression vectors. Detected proteins are indicated by arrows. The quantities of SOX18 protein in cells overexpressing EGR1 were calculated as a percentage of mock transfected cells which was set as $100 \%$. Data of three (HeLa) or two (EA.hy926) independent experiments are presented at histograms as the means \pm SEM. Values of $P$ $<0.05$ are presented by* terpart to drive the expression of reporter gene was analyzed in both HeLa and EA.hy926 cell lines. Mutations in EGR1 binding sites reduced promoter activity to $56 \%$ and $14 \%$ in HeLa and EA.hy926 cell lines, respectively (Figure $5 \mathrm{~B}$ ). These results indicate that EGR1 binding sites clustered immediately upstream of $t s p$ are of functional relevance for the activity of SOX18 minimal promoter region, while the remarkable effect observed in EA.hy926 cells could indicate the vital importance of this control element in the regulation of SOX18 gene expression in endothelial system.

\section{EGR1 overexpression increases SOX18 mRNA and protein levels}

We have performed real time RT-PCR and western blot analysis in order to determine the effect of EGR1 overexpression on SOX18 mRNA and protein levels in HeLa and EA.hy926 cells. Quantitative real time RT-PCR has demonstrated that EGR1 overexpression leads to increase in SOX18 mRNA level and leads to induction of SOX18 gene transcription by approximately 1.5 - and 1.8 -fold in
HeLa and EA.hy926 cells, respectively (Figure 6A). Importantly, 1.4- and 1.3-fold increase in SOX18 protein level was also detected in HeLa and EA. hy926 cells, respectively (Figure $6 \mathrm{~B}$ ). These results signify the functional importance of EGR1 in activation of SOX18 gene expression in a native context.

\section{Discussion}

Transcription factors serve as master switches for regulating a number of developmental processes (Oettgen, 2001). Along with others, transcription factor SOX18 is shown to be involved in regulation of vascular development and postnatal neovascularization (Downes and Koopman, 2001). It has been demonstrated that SOX18 participates in the VEGF-FIk1 pathway of endothelial cells activation (Darby et al., 2001). Although three SOX18 target genes have been identified so far (Im et al., 2001; Hosking et al., 2004; Fontijn et al., 2008) and the role of SOX18 in angiogenesis is established (Young et al., 2006), little is known regarding its 
transcriptional regulation. Accordingly, the aim of this study was to carry out comprehensive functional characterization of the human SOX18 promoter, including determination of transcription start point and identification of control elements involved in the regulation of SOX18 gene expression, with an emphasis on angiogenesis-related transcription factors.

Our in silico analysis of putative transcription factor binding sites within SOX18 promoter region, among other transcription factors, revealed six putative EGR1 binding sites. By in vitro binding assays and functional analyses we have shown that three putative binding sites, located in a cluster close to $t s p$, are functionally relevant and sufficient for EGR1-induced SOX18 transcription. It is common for GC-rich promoter regions of genes such as SOX18 to possess several binding sites for transcription factors belonging to EGR family that often overlap with the ones for other zinc-finger transcription factors such as specificity protein 1 (Sp1) and Wilms tumor 1 (WT1) (Silverman and Collins, 1999). In vitro studies suggest that these transcription factors can displace one another from many promoters depending on equilibrium determined by their concentration and binding affinity (Silverman and Collins, 1999).

Previously, we have shown that zinc-finger transcription factors Sp3 and ZBP-89 are capable of binding to overlapping sites within SOX18 promoter region (Petrovic et al., 2009). We have shown that these proteins act as down-regulators of SOX18 promoter activity (Petrovic et al., 2009). Here, we have presented up-regulation of SOX18 promoter by EGR1, acting through the same overlapping region. EGR1 consensus sequence often overlap with $\mathrm{Sp} 1 / \mathrm{Sp} 3$ binding sites in many EGR1 target genes (Akuzawa et al., 2000). It has been reported that EGR1 binds to an element overlapping the Sp1 site in the PDGF-B promoter (Khachigian et al., 1996). In these studies, Sp1 contributes to basal level of gene expression, whereas EGR1 functions as an inducible transcription factor, activated in response to vascular injury, displacing pre-bound Sp1 (Khachigian et al., 1996). In this report we have demonstrated that EGR1 overexpression overcomes Sp3-mediated repression of SOX18 promoter activity. Presented results indicate that competition between members of $\mathrm{Sp}$ and EGR protein families might play an important role in the regulation of SOX18 gene expression. We would like to hypothesize that SOX18 up- or down-regulation depends on the concentration of these proteins in the nucleus, probably governed by different stimuli. It was previously shown that EGR1 expression level is low in quiescent endothelial cells, but is dramatically increased upon exposure to different stimuli such as hypoxia, tissue injury and mechanical stress (Akai et al., 1994; Khachigian et al., 1996; Yan et al., 1999). Similarly, Sox18 expression is up-regulated after injurious stimuli in endothelial cells during wound healing and tissue repair (Darby et al., 2001). Taken together, it is likely that in quiescent endothelium, SOX18 basal transcription is governed by other GC-binding transcription factors, like $\mathrm{Sp}$-family members, while upon stimulation, EGR1 acts as trans-activator of SOX18 transcription. It is important to point out that the same mechanism has been previously reported and described in vascular systems (Silverman and Collins, 1999).

Here we present that EGR1 overexpression leads to the up-regulation of SOX18 promoter activity in both tumor (HeLa) and endothelial (EA.hy926) cell line. However, we have observed the difference in the response of minimal promoter region construct between two cell lines. While $5^{\prime}$ deletion of full length promoter up to -89 (construct 255pCAT6) did not cause any significant change in the response to overexpressed EGR1 in HeLa cells, this deletion led to decreased response in EA.hy926 cell line (Figures 4B and 4C). Nevertheless, mutations of clustered EGR1 binding sites within SOX18 minimal promoter region remarkably impair promoter activity, particularly in EA.hy926 cells (Figure 5B), signifying the major importance of this regulatory region in EGR1-mediated activation of SOX18 gene transcription in endothelial setting.

For both SOX18 and EGR1 it has been shown to play important roles in angiogenesis and tumor growth (Lucerna et al., 2006; Young et al., 2006). Significant expression of these transcription factors has been found during wound healing (Khachigian et al., 1996; Darby et al., 2001) and in atherosclerotic lesions (McCaffrey et al., 2000). By data presented in this study, we have established SOX18 as a novel target gene regulated by EGR1 transcription factor. Besides SOX18 promoter reported here, EGR1 binding elements have been identified in the promoters of several other genes involved in angiogenic processes including growth factors (fibroblast growth factor- 2 and TGF- $\beta$ ), adhesion molecules (intercellular adhesion molecule 1) and cytokines (TNF $\alpha$ ) (Kim et al., 1989; Biesiada et al., 1996).

Angiogenesis induced by factors produced in cancer cells has been recognized as a critical step in tumor growth, progression and methastasis (Carmeliet, 2005). Since recent research demonstrated that SOX18 is expressed during initial steps 
of tumor vascularization, this gene has already been recognized as potential target for anti-angiogenic therapy (Young et al., 2006). In parallel, other studies provided evidence for important role of EGR1 in microvascular endothelial cell growth, neovascularization, tumor angiogenesis and tumor growth (Fahmy et al., 2003), identifying this protein as an another potential therapeutic target. In this study, for the first time, we have provided a functional link between SOX18 and EGR1, two potential targets in anti-angiogenic therapy. Thus, clarifying the complex mechanisms involved in transcriptional regulation of human SOX18 gene could help in better understanding of important physiological and patho-physiological processes in which SOX18 gene participates.

Taken together, we have determined unique SOX18 transcription start point, defined minimal promoter region indispensable for SOX18 transcription and revealed the presence of essential regulatory element(s) positioned between -89 and +29 relative to tsp. Furthermore, by functional analyses and site directed mutagenesis we have demonstrated the important role of EGR1 in the up-regulation of SOX18 promoter activity. Finally, we have shown that EGR1 up-regulates SOX18 expression in a native context on both mRNA and protein level. We believe that presented results provide an important contribution in understanding of SOX18 gene transcriptional regulation, since this gene is marked as a potential anti-angiogenic target.

\section{Methods}

\section{Cell culture}

HeLa (ATCC CCL 2) were maintained in DMEM supplemented with $10 \%$ FBS and $1 \times$ MEM nonessential amino acids at $37^{\circ} \mathrm{C}$ in $5 \% \mathrm{CO}_{2}$. EA.hy926 cells (Edgell et al., 1983) were maintained in DMEM, supplemented with $10 \%$ FBS and hypoxanthine/aminopterin/thymidine (HAT supplement) at $37^{\circ} \mathrm{C}$ in $5 \% \mathrm{CO}_{2}$.

\section{Primer extension analysis}

Antisense primers used in primer extension reactions were: PE5: 5'-TCGTCCTGTGCTCCGTAGCCGGGCGGC-3' (+35), PE3: 5'-AAGGGCAGGCCAGGCCGGGAGGGCGGATG-3' (-78).

The numbers indicated in parenthesis correspond to the distance in nucleotides from 5' end of the sequence to the A of the first ATG. Primers were end labeled with $\left[\gamma^{32} \mathrm{P}\right]$ ATP. $20 \mu \mathrm{g}$ of total cellular RNA prepared either from EA.hy926 or HeLa cells (Rnase mini Kit, QIAGEN) was mixed with radiolabeled primer $\left(10^{5} \mathrm{cpm}\right)$ and incubated at $65^{\circ} \mathrm{C}$ for $5 \mathrm{~min}$. The annealing mix was cooled on ice, and primers were extended with 200 units of SuperScript ${ }^{\mathrm{TM}}$ RnaseH-reverse transcriptase (Invitrogen) for $1 \mathrm{~h}$ at $37^{\circ} \mathrm{C}$ in a reaction containing 30 units of RNAguard ${ }^{\mathrm{TM}}$ (Amersham Pharmacia Biotech), $2 \mathrm{mM}$ dNTP mix, $10 \mathrm{mM}$ DTT, $50 \mathrm{mM}$ Tris- $\mathrm{HCl}(\mathrm{pH} 8.3), 75 \mathrm{mM} \mathrm{KCl}$ and $3 \mathrm{mM} \mathrm{MgCl}_{2}$. Control reaction was performed with $10 \mu \mathrm{g}$ of yeast tRNA. The lengths of the primer extension products were determined by comparison to dideoxy-sequencing reaction performed using the same primers (T7 Sequencing Kit, USB) as well as to the radiolabeled PhiX/HinflII DNA ladder.

\section{Generation of SOX18 promoter constructs}

SOX18 promoter fragments Pstl-Pvull (892 bp), Pstl-Stul (637 bp), Stul-Pvull (255 bp) and Narl-Pvull (137 bp) were first cloned in pBSKS+ vector. Fragments were subsequently released by HindlII-BamHI, except for the fragment Narl-Pvull that was released by Sall-BamHI, and cloned in pBLCAT6 to generate clones 892pCAT6, 637pCAT6, 255pCAT6, and 137pCAT6. Mutated fragment 255mut was commercially synthesized (GeneScript Corporation), cloned into pUC57 vector and subsequently subcloned into pBLCAT6 vector in HindIII/BamHI sites.

\section{In silico analysis of the SOX18 $5^{\prime}$ upstream regulatory region}

MatInspector Release professional 7.2.2 (http://www.genomatix.de/) was used to analyze putative transcription factor binding sites within the SOX18 promoter region.

\section{Transient transfection assays}

HeLa cells were transfected using calcium-phosphate precipitation method. $1.2 \times 10^{6}$ cells were seeded in $10 \mathrm{~cm}$ dish (two dishes per transfection) and transfected with 10 $\mu \mathrm{g}$ of various SOX18 promoter constructs, together with 3 $\mu \mathrm{g}$ of $\mathrm{pCH} 110$ vector (Amersham Pharmacia Biotech) and $4 \mu \mathrm{g}$ of pBluescript (Stratagene). In co-transfection assays $2 \mu \mathrm{g}$ of either empty pcDNA3.1 or pcDNA3.1-EGR1 expression vector was used. EA.hy926 cells were transfected using LIPOFECTAMINE reagent (Invitrogen). For each transfection, $2 \times 10^{6}$ cells were seeded into $6-\mathrm{cm}$ dish and $4.5 \mu \mathrm{g}$ of various SOX18 promoter constructs together with $1.5 \mu \mathrm{g}$ of $\mathrm{pCH} 110$ vector were mixed with $16 \mu \mathrm{l}$ of LIPOFECTAMINE reagent. In co-transfection assays, 0.8 $\mu \mathrm{g}$ of either empty pcDNA3.1 or pcDNA3.1-EGR1 expression vector was used. In functional competition assays, both HeLa and EA.hy926 cells were co-transfected with fixed amount of Sp3 expression vector $(4 \mu \mathrm{g}$ and $0.8 \mu \mathrm{g}$, respectively) and increasing amounts of EGR1 expression vector, where Sp3:EGR1 ratios were 1:0.5 and 1:1. Extracts for $\beta$ galactosidase and chloramphenicol acetyltransferase (CAT) assays were prepared after $24 \mathrm{~h}$ and $48 \mathrm{~h}$ for EA.hy926 and HeLa cells, respectively. $\beta$ galactosidase and CAT assays were performed as described (Kovacevic Grujicic et al., 2005). The normalized CAT activities were evaluated as a percentage of the selected promoter construct which was set as $100 \%$ activity. Mean values of relative CAT activities were compared with Student's $t$ test using SPSS software. A 
$P$-value of less than 0.05 was considered significant.

\section{Nuclear extract preparation and electrophoretic mobility shift assays (EMSA)}

Nuclear extracts from HeLa cells were prepared according to standard procedure (Dignam et al., 1983). The following sense oligonucleotides were used in the EMSA and supershift studies: EGR probe I: 5'-CAAGGGCCCTTGGGGGGCAGGGAGGACG 3' (-665), EGR probell: 5'-GAGCCTCCCAGCGGGGGGCGGGGAACGGCAA 3' (-303), EGR probe III: 5'-GGGGGAGGTGGGGGGGCTGTGCGC GGGGGAGG 3' (-121), EGR probe IV: 5'-GACCCGCCCCCGCCCGCCCGCCCCGCCGCCGATTGG 3' (-29), Consensus EGR1: 5'-GGATCCAGCGGGGGCGAGCGGGGGCGA 3'.

The numbers indicated in parenthesis correspond to the distance in nucleotides from 5 ' end of the sequence to the transcription start point $(t s p)$. All antisense oligonucleotides were designed to contain $2 \sim 3$ unpaired $G$ nucleotides at $5^{\prime}$ end and probes were labeled by fill-in reaction. EMSA experiments were performed as described (Kovacevic Grujicic et al., 2005). For supershift assay, EMSA reaction was preincubated for $30 \mathrm{~min}$ at $37^{\circ} \mathrm{C}$ with $2 \mu \mathrm{g}$ of antiEGR1 antibody (Cell Signaling Technology, \#4154).

\section{Western blot analysis}

Whole cell lysates (WCL) were prepared after transient transfection of HeLa and EA.hy926 cells with either empty pcDNA3.1 or pcDNA3.1-EGR1 expression vectors. Cells were collected and lysed with lysis buffer containing 50 $\mathrm{mM}$ Tris-Cl pH 8.0, $150 \mathrm{mM} \mathrm{NaCl}, 1 \% \mathrm{NP}-40,5 \mu \mathrm{g} / \mathrm{ml}$ PMSF, $1 \mu \mathrm{g} / \mathrm{ml}$ aprotinin, $1 \mu \mathrm{g} / \mathrm{ml}$ leupeptin and $1 \mu \mathrm{g} / \mathrm{ml}$ pepstatin. Proteins were separated on $10 \%$ SDS-PAGE. Membranes were blocked in $10 \%$ non-fat milk for $1 \mathrm{~h}$, following the incubation with anti-SOX18 antibody (sc20100, Santa Cruz Biotechnology) for $1 \mathrm{~h}$ at room temperature. Mouse anti- $\alpha$-tubulin Ab (CP06 - Calbiochem) was used for normalization of protein amount. Immunoreactive bands were detected using HRP-conjugated antirabbit IgG (Amersham Pharmacia Biotech) or anti-mouse IgG (Amersham Pharmacia Biotech) and ECL detection kit (Amersham Pharmacia Biotech). Immunoblots were digitalized and quantified with ImageQuant Version 5.2 software and normalized for $\alpha$-tubulin values. The quantities of SOX18 protein were calculated as a percentage of mock transfected cells which was set as $100 \%$. Mean values of relative SOX18 expression were compared with Student's $t$-test using SPSS software. A $P$-value of less than 0.05 was considered significant.

\section{Two-step qRT-PCR}

Total RNAs from HeLa and EA.hy926 cells, either mock transfected or transfected with EGR1 expression construct, were isolated using TRI-Reagent (Ambion) and $1 \mu \mathrm{g}$ of each RNA was reverse transcribed using MuLV reverse transcriptase (Applied Biosystems) and random hexamers (Applied Biosystems). cDNAs were subjected to real time PCR using Power SYBR Green PCR Master Mix (Applied
Biosystems) in 7500 Real-Time PCR Systems (Applied Biosystems). SOX18 cDNA was amplified using following primers: forward, 5' TTCCATGTCACAGCCCCCTAG 3', and reverse 5' GACACGTGGGAACTCCAG 3'. GAPDH used as endogenous control was amplified using following primers: forward, 5' GGACCTGACCTGCCGTCTAG 3' and reverse, 5' CCACCACCCTGTTGCTGTAG 3'. All samples were measured in triplicates and the mean value was considered. The relative level of SOX18 expression was determined using comparative quantification algorithm where resulting $\Delta \Delta \mathrm{Ct}$ value was incorporated to determine the fold difference in expression $\left(2^{-\Delta \Delta C t}\right)$. Relative SOX18 expression was presented as percentage of SOX18 expression in mock transfected cells that was set as $100 \%$. Values were compared with Student's $t$ test using SPSS software and $P$-value of less than 0.05 was considered significant.

\section{Acknowledgements}

This work has been supported by Ministry of Science and Technological Development, Republic of Serbia (Grant No. 143028) and by the International Centre for Genetic Engineering and Biotechnology (Grant No. CRP/YUG 07-01). We thank Prof Cora Jean Edgell for the generous gift of EA.hy926 cell line and Dr Sarki A. Abdulkadir, for expression vector pcDNA3.1-EGR1.

\section{References}

Akai Y, Homma T, Burns KD, Yasuda T, Badr KF, Harris RC. Mechanical stretch/relaxation of cultured rat mesangial cells induces protooncogenes and cyclooxygenase. Am J Physiol 1994;267:C482-90

Akuzawa N, Kurabayashi M, Ohyama Y, Arai M, Nagai R. Zinc finger transcription factor Egr-1 activates Flt-1 gene expression in THP-1 cells on induction for macrophage differentiation. Arterioscler Thromb Vasc Biol 2000;20: 377-84

Argyropoulos G, Rankinen T, Bai F, Rice T, Province MA, Leon AS, Skinner JS, Wilmore JH, Rao DC, Bouchard C. The agouti-related protein and body fatness in humans. Int $\mathrm{J}$ Obes Relat Metab Disord 2003;27:276-80

Biesiada E, Razandi M, Levin ER. Egr-1 activates basic fibroblast growth factor transcription. Mechanistic implications for astrocyte proliferation. J Biol Chem 1996;271: 18576-81

Bowles J, Schepers G, Koopman P. Phylogeny of the SOX family of developmental transcription factors based on sequence and structural indicators. Dev Biol 2000;227: 239-55

Carmeliet P. VEGF as a key mediator of angiogenesis in cancer. Oncology 2005;69 Suppl 3:4-10

Cermenati S, Moleri S, Cimbro S, Corti P, Del Giacco L, Amodeo R, Dejana E, Koopman P, Cotelli F, Beltrame M. Sox18 and Sox7 play redundant roles in vascular development. Blood 2008;111:2657-66

Darby IA, Bisucci T, Raghoenath S, Olsson J, Muscat GE, 
Koopman P. Sox18 is transiently expressed during angiogenesis in granulation tissue of skin wounds with an identical expression pattern to Flk-1 mRNA. Lab Invest 2001;81:93743

Dignam JD, Lebovitz RM, Roeder RG. Accurate transcription initiation by RNA polymerase II in a soluble extract from isolated mammalian nuclei. Nucleic Acids Res 1983;11: 1475-89

Downes M, Koopman P. SOX18 and the transcriptional regulation of blood vessel development. Trends Cardiovasc Med 2001;11:318-24

Edgell CJ, McDonald CC, Graham JB. Permanent cell line expressing human factor VIII-related antigen established by hybridization. Proc Natl Acad Sci USA 1983;80:3734-7

Fahmy RG, Dass CR, Sun LQ, Chesterman CN, Khachigian LM. Transcription factor Egr-1 supports FGF-dependent angiogenesis during neovascularization and tumor growth. Nat Med 2003;9:1026-32

Fontijn RD, Volger OL, Fledderus JO, Reijerkerk A, de Vries $\mathrm{HE}$, Horrevoets AJ. SOX-18 controls endothelial-specific claudin- 5 gene expression and barrier function. Am J Physiol Heart Circ Physiol 2008;294:H891-900

Garcia-Ramirez M, Martinez-Gonzalez J, Juan-Babot JO, Rodriguez C, Badimon L. Transcription factor SOX18 is expressed in human coronary atherosclerotic lesions and regulates DNA synthesis and vascular cell growth. Arterioscler Thromb Vasc Biol 2005;25:2398- 403

Hosking BM, Wang SC, Downes M, Koopman P, Muscat GE. The VCAM-1 gene that encodes the vascular cell adhesion molecule is a target of the Sry-related high mobility group box gene, Sox18. J Biol Chem 2004;279:5314-22

Im HJ, Smirnov D, Yuhi T, Raghavan S, Olsson JE, Muscat GE, Koopman P, Loh HH. Transcriptional modulation of mouse mu-opioid receptor distal promoter activity by Sox 18 . Mol Pharmacol 2001;59:1486-96

Irrthum A, Devriendt K, Chitayat D, Matthijs G, Glade C, Steijlen PM, Fryns JP, Van Steensel MA, Vikkula M. Mutations in the transcription factor gene SOX18 underlie recessive and dominant forms of hypotrichosis-lymphedema-telangiectasia. Am J Hum Genet 2003;72:1470-8

Khachigian LM, Lindner V, Williams AJ, Collins T. Egr-1induced endothelial gene expression: a common theme in vascular injury. Science 1996;271:1427-31

Kim SJ, Jeang KT, Glick AB, Sporn MB, Roberts AB. Promoter sequences of the human transforming growth factorbeta 1 gene responsive to transforming growth factor-beta 1 autoinduction. J Biol Chem 1989;264:7041-5

Kovacevic Grujicic N, Mojsin M, Krstic A, Stevanovic M.
Functional characterization of the human SOX3 promoter: identification of transcription factors implicated in basal promoter activity. Gene 2005;344:287-97

Lucerna M, Mechtcheriakova D, Kadl A, Schabbauer G, Schafer R, Gruber F, Koshelnick Y, Muller HD, Issbrucker K, Clauss M, Binder BR, Hofer E. NAB2, a corepressor of EGR-1, inhibits vascular endothelial growth factor-mediated gene induction and angiogenic responses of endothelial cells. J Biol Chem 2003;278:11433-40

Lucerna M, Pomyje J, Mechtcheriakova D, Kadl A, Gruber F, Bilban M, Sobanov Y, Schabbauer G, Breuss J, Wagner O, Bischoff M, Clauss M, Binder BR, Hofer E. Sustained expression of early growth response protein-1 blocks angiogenesis and tumor growth. Cancer Res 2006;66:670813

Matsui T, Kanai-Azuma M, Hara K, Matoba S, Hiramatsu R, Kawakami H, Kurohmaru M, Koopman P, Kanai Y. Redundant roles of Sox17 and Sox18 in postnatal angiogenesis in mice. J Cell Sci 2006;119:3513-26

McCaffrey TA, Fu C, Du B, Eksinar S, Kent KC, Bush H, Jr., Kreiger K, Rosengart T, Cybulsky MI, Silverman ES, Collins T. High-level expression of Egr-1 and Egr-1-inducible genes in mouse and human atherosclerosis. J Clin Invest 2000; 105:653-62

Oettgen P. Transcriptional regulation of vascular development. Circ Res 2001;89:380-8

Petrovic I, Stevanovic M. The human SOX18 gene: expression analysis and characterization af Its 5 ' flanking region. Arch Biol Sci 2007;59:267-72

Petrovic I, Kovacevic-Grujicic N, Stevanovic M. ZBP-89 and Sp3 down-regulate while NF-Y up-regulates SOX18 promoter activity in HeLa cells. Mol Biol Rep 2009;36:993-1000

Pevny LH, Lovell-Badge R. Sox genes find their feet. Curr Opin Genet Dev 1997;7:338-44

Silverman ES, Collins T. Pathways of Egr-1-mediated gene transcription in vascular biology. Am J Pathol 1999;154:66570

Wegner M. From head to toes: the multiple facets of Sox proteins. Nucleic Acids Res 1999;27:1409-20

Yan SF, Mackman N, Kisiel W, Stern DM, Pinsky DJ. Hypoxia/Hypoxemia-Induced activation of the procoagulant pathways and the pathogenesis of ischemia-associated thrombosis. Arterioscler Thromb Vasc Biol 1999;19:2029-35

Young N, Hahn CN, Poh A, Dong C, Wilhelm D, Olsson J, Muscat GE, Parsons P, Gamble JR, Koopman P. Effect of disrupted SOX18 transcription factor function on tumor growth, vascularization, and endothelial development. J Natl Cancer Inst 2006;98:1060-7 